\title{
ELECTROMYOGRAMS IN MUSCULAR DISORDERS
}

\author{
BY \\ ERIC KUGELBERG \\ From the Neurological Clinic Serafimerlasarettet, Stockholm \\ (Received 18Th July, 1947)
}

\section{Introduction}

IN the routine work at this clinic during the last few years, electromyography has been found a reliable method for distinguishing between muscular dystrophies and lesions of the lower motor neurone. When other clinical methods have failed it has been possible by the aid of electromyography to differentiate not only between late distal forms of myopathy from peroneal muscular atrophy, but also proximal forms of muscular dystrophy from certain cases of proximal spinal muscular atrophy.

There are but few published observations on the electromyogram in cases of muscular dystrophy in which the modern technique introduced by Adrian and Bronk (1929) with coaxial needle electrodes has been applied. Buchthal and Clemmesen (1941 a) used this method in order to differentiate muscular dystrophy from neurogenic lesions. In genuine severe myopathy they found, as in the normal muscle, that voluntary contraction was accompanied by the interfering activity of many motor units. Only in extremely severe atrophy (observed in one muscle) did they note a slight decrease in the number of active units, as is seen in cases of mild injury of the lower motor neurone. In moderate or severe neurogenic lešions, on the other hand, the number of active units registered is markedly reduced. On maximal contraction, " single discharges" may be obtained without interference from neighbouring units, as had also been observed by Seyffarth (1938, 1940) and by Weddell and others (1944).

Bòwden and Gutmann (1946) recorded the electrical activity of muscle in one case of progressive muscular dystrophy. Here, however, on maximal contraction merely a few action potentials were left, some of which were indistinguishable from action potentials characteristic of fibrillation.

The present paper is concerned mainly with a study of the changes in the electrical activity of voluntary muscle in man in various forms of myopathy. For the purpose of comparison, electromyographic changes in different types of lesions of the lower motor neurone are described.

\section{Technique}

Coaxial needle electrodes (platinum wire, $0.20 \mathrm{~mm}$ ? in diameter, insulated by a glass capillary tube from cannula of stainless steel with an outer diameter of $0.5 \mathrm{~mm}$.) were employed. The action potentials were led off to a one-channel balanced condenser-coupled amplifier connected to one beam of a cathode-ray tube $\stackrel{\infty}{2}$ the other beam of the tube was connected to an oscillator which recorded the time in milliseconds or $1 / 100$ secondiThe time constant was about $1 / 30$ of a second, and the gain linear between $20-4,000$ cycles per second.

For diagnostic purposes, it is sufficient to observe the picture on the screen. Only if the duration and size of the action potentials are to be exactly determined wilb measurements from tracings be necessary. In this studjic the picture on the screen was first observed at different sweep velocities. In order to obtain surveys of the course of the voluntary contraction, the sweep then stopped and a picture was taken while the patient was making a minimal continuous contraction, sla increasing to maximal. The speed at which the phatoo graphic paper was fed forward was $10 \mathrm{~cm}$. per secood

In order to obtain detailed pictures of individtal action potentials, the cathode ray tube was rotated 900 degrees, so that the sweep carried the ray horizontanly over the screen about three times a second. When the film is running vertically in front of the tube, a series op such pictures one above the other is obtained. Wits the sweep velocity adopted, $\frac{1}{4}$ to $1 \mathrm{~cm}$. on the screen corresponded to a millisecond, which permits an exach determination of the duration and form of the actior potentials.

\section{Results \\ Primary Muscular Disorders}

In the muscles under examination, the patient was asked to make a minimal "contraction, slowlys increasing in intensity, so that four or five differen " units," recorded with the needle at the samer position, could be followed from the start untiß. they had merged into the activity of neighbouring " units." The position of the needle was then changed. In this way, at several positions in thes muscle, samples of action potentials were available. for quantitative determinations of both duration and amplitude. At the same time one couls. obtain an idea as to how many active units theres were on maximal contraction. 
The position of the needle is carefully tested under control of the picture on the screen. In large muscles it may often be difficult to separate the different " units." Sometimes, as found by Gilson and Mills (1941), one succeeds best with the point of the needle immediately under the fascia. The small intrinsic muscles of the hand and foot are the most easily examined.

The finding of a suitable position for the needle is largely a matter of chance. In the muscle there is a functional organization of different units, certain of them being activated by a slight contraction, others only as the contraction increases in intensity. The aim is, therefore, to bring the point of the needle in close contact with a low-threshold unit which can be recorded without disturbance. The point of the needle should not lie outside the muscle, nor in areas which are silent owing to pathological changes.

An unsuitably placed needle electrode gives no more information than can be recorded with pad electrodes, as exemplified by Fig. 1. This record is taken from a severe case of progressive muscular dystrophy (Case 1) from the greatly atrophied first interosseus muscle. Contractile tissue could scarcely be palpated, and there were no visible movements of the fingers when an attempt was made to spread them out. The needle position was first adjusted so as to obtain isolation of the action potentials (record a), of which a detailed picture is presented in record $d$. The number of units was greatly reduced. Only two or three are repetitive, with a frequency of 30 per second and occasionally up to 50 during maximal contraction. The high frequency is a good check that the person examined is doing his best to contract the muscle.

If (record b) the position of the needle is gently shifted a few millimetres, the base line becomes disturbed. The fine, distinct action potentials which repeat with a fairly constant amplitude are to an increasing degree replaced, even in minimal contraction, by broad, irregular potentials. If the patient makes a maximal contraction, they may have a rather large amplitude (Fig. 1c). This interfering activity fails to give the information that the muscle examined has a greatly reduced number of active units (Fig. 1a), as well as rapidly passing spikes with a duration of about one millisecond (Fig. 1d), and is therefore valueless for the purpose of differential diagnosis. Unless this fallacy is recognized one is liable to draw erroneous conclusions, for if the needle is not properly placed this same type of interfering activity may be seen also in the normal muscle and in cases of severe neurogenic lesions even on weak contraction.
Progressive Muscular Dystrophy.-Commencing in the most advanced stages of progressive muscular dystrophy a wasted muscle may appear to be electrically silent. If it is systematically searched, however, islands with greatly reduced activity are usually found, a few action potentials being brought into operation on maximal contraction (Fig. 1a). The spikes then have a very short duration, about 1 millisecond (Fig. 1d), that is, they are nearly as rapid as a motor nerve fibre spike potential. A few broader spikes may, however, remain. The occurrence of a relatively large number of very rapid action potentials is the most characteristic and constant change in the dystrophic muscle.

If the pathological process is somewhat less severe, several active units will be left which are sufficiently numerous to disturb the whole base line, although the largest spikes can still be followed separately in maximal contractions (Figs. 2 and 3).

Fig. 2 from the biceps muscle (Case 5) shows a voluntary contraction gradually increasing in strength. The activity starts with a small spike having an amplitude of 50 microvolts and a frequency of 14 per second (record a).- The following three or four units (Fig. 2b) have an amplitude that must be regarded as pathologically small, that is $\mathbf{5 0}$ to 100 microvolts. In a normal biceps the units recruited as Nos. 2 to 4 would have had an amplitude of at least 100 to 500 microvolts. These small spikes all have a duration of about 1 millisecond. They are evidently identical with the action potentials which are indistinguishable from fibrillary spikes as reported in Bowden and Gutmann's case. The next unit recruited (record b) has an amplitude of 400 microvolts and a duration of 1 millisecond (record d). It can be followed on maximal contraction (record c), in spite of the interfering activity on the base line. The maximum frequency attained is 60 per second. The biceps muscle examined was greatly atrophied, about $1 \mathrm{~cm}$. in diameter, and markedly paretic, though the degree of paresis could not be exactly determined, as the brachialis anticus was relatively intact.

The activity recorded in Case 4 (Fig. 3) shows the same general features. There are no abnormally small spikes, but all have a duration of 1 to 2 milliseconds (record c). On maximal contraction (record b) the action potentials only just take up the whole base line; six or seven of them are recruited at the maximum frequency of 45 per second. This record is from the right quadriceps muscle. The patient, when sitting on a chair with the legs straight, could not lift the heel from the floor. A normal quadriceps would have exerted considerably greater force in a case where the whole 
- base line on the electromyogram had been taken up by action potentials, which indicates that the individual "units" represented by the spikes had been reduced in strength.

- When a muscle is strong enough to perform a movement at the joint against weak resistance, the number of recorded units on maximal contraction generally appears to be normal (Fig. 4), so that with moderate contraction they cover the whole base line (record c). Thus, the number of spikes in the electromyogram is not a sensitive-index of changes in the muscle -due to dystrophy. The majority of the spikes, however, still have a duration of 1 to 2 milliseconds (records $d$ and e). These rapid spikes are often numerous in muscles showing only a slight paresis and atrophy : thus, in a record from a biceps muscle (Fig. 5, Case 3) they numbered about 40 or 50 per cent. of all the spikes registered. This, however, is not a constant finding.

It is often difficult to see any pathological features in the electromyogram from a slightly paretic muscle in cases of muscular dystrophy. Moreover, as shown by Snodgrass and Sperry (1941), in the extensor digitorium communis there are action potentials in the normal muscle of very short duration. The occurrence of spike potentials with a duration of 1 to 2 milliseconds is thus not always a pathological feature. Normally however, they occur sparsely, except in the facial muscles, where they are relatively common. In normal muscle they have to be searched for among the majority of spikes which have a duration of 4 to 10 milliseconds.

In three cases out of six in this group there was such a marked reduction in the number of active " units" that "single discharges" were obtained on maximal voluntary contraction.

Still more often one can find " single discharges" in the relatively benign forms of muscle dystrophy where the pathological process is confined to a few muscle groups. In such cases the changes develop during a much longer period than in the malignant generalized forms of dystrophy. This is seen in three examples of the facio-scapulo-humeral type of Landouzy and Dejerine (1884) (Cases 7-9), and in one case of the scapulo-humeral form of Landouzy and Dejerine (1886) (Case 10), where the disease had been confined for fifteen years or more to the shoulder-girdle, the thigh, and the anterior tibial muscle groups.

In the anterior tibial muscles of these cases only a few action potentials were recruited on maximal contraction. In this respect the picture was of the type which hitherto has been considered characteristic of neurogenic lesions. The spikes however were only of about 1 millisecond's duration.
Late Distal Hereditary Myopathy.-This applies also to the following group which deserves a more detailed description. Cases 11 to 25 are examples of a late distal hereditary myopathy, a type briefly described by Welander (1946), whose cases I have of had the opportunity of examining. A complete description of this disease is in the course of publica- 0 tion by Welander. The disease closely resembles peroneal muscular atrophy. It is characterized by a late onset. The muscles earliest and most severely $\underset{\overrightarrow{0}}{\overrightarrow{0}}$ involved are the intrinsic muscles of the hand; next in order follow the extensors on the forearm, $\overrightarrow{\overline{\vec{N}}}$ the short extensors of the toes, and the tibial and peroneal groups, the peroneal being usually less affected than the tibial. Mild subjective sensory disturbances occurring distally in the extremities were observed in one out of the fifteen cases 2 examined. The progress of the disease is very slow. is The late onset, at an age between 35 and 60 years, $\vec{\circ}$ beginning in the upper extremities, as well as the absence of cramp, fascicular twitching, and more $\vec{\omega}$ pronounced sensory disturbance, make it possible $\frac{\rho}{\sqrt{3}}$ to differentiate the disease, with tolerable certainty, from the Charcot-Marie-Tooth type of muscular atrophy.

Distal myopathies of a somewhat different type $\frac{\omega}{N}$ have also been described by Milhorat and WoG $\mathrm{T}$ (1943).

In some of these cases the insertion of the needfe was followed by a series of action potentials of the fibrillary type, which died away in a few seconds, and indicated an increased mechanical irritabilits? When voluntary activity was already lost in tie muscle to be tested, "insertion activity" coufd not be obtained, this being, in contrast to the findings in neurogenic paralysis, where spontaneous and mechanical irritability may remain for many years after denervation of the muscle. It seems that in myopathies the mechanical responses dis- $\frac{\circ}{\mathbb{Q}}$ appear simultaneously with voluntary activity.

"Single discharges" were found in seven or eight of the fifteen cases examined (Figs. 6 and 7). In Fig. 6, from the anterior tibial muscle (Case 12), only two different spikes were recruited on maximal contraction. The larger one had a frequency of $\bar{D}$ 30 to 50 per second and an amplitude of 700 microvolts (record a), and its duration was 1 millisecond $\frac{5}{3}$ (record b). The smaller unit could be detected only if the amplification was increased (record c). Its $\frac{3}{3}$ amplitude was pathologically small, being merely 25 microvolts, and its duration 1 millisecond.

In Fig. 7 from the short extensor of the toes $\frac{7}{0}$ (Case 14) 3 or 4 different " units" were recruited. Records $a$ and $b$ show the instability of the spike $N$ amplitude, so characteristic of these rapid spikes. N 
On voluntary contraction, the amplitude of the largest spike diminished rapidly, but by degrees, from 1.5 millivolts to 500 microvolts. The reason was presumably that the point of the needle had shifted, but a blocking of some fibres in the " unit" would have produced the same result (Hoefer and Putnam, 1939 ; Buchthal and Clemmesen, 1941 a).

As shown in Fig. 8 (from the first interosseus muscle of Case 12), the number of units is larger in the less affected muscles. Records a, b, c show the recruitment of spikes with an increasing amplitude as the contraction is intensified. The contraction is introduced by a small spike of 50 microvolts, followed in turn by units of 100 and 200 microvolts (record a); next comes one of 250 microvolts (record b), and finally a spike of 800 microvolts (record c). Thereafter the recruiting cannot be followed any further. All the action potentials have a duration of 1 to 2.5 milliseconds (record e). Although a considerable number of units were mobilized on maximal voluntary contraction (record d), the patient could barely move the second finger. In this case, therefore, the individual action potentials represent very much less muscle strength than in normal muscle. The same condition is exemplified by Fig. 9 from the first interosseus muscle (Case 15). Records $b$ and $c$ show the activity on maximal contraction at two different positions of the needle while the strength developed by the muscle barely sufficed to move the forefinger.

For the purpose of comparison with neurogenic lesions, the amplitudes of the action potentials were measured in the order in which they were "recruited" (see Table) as the contraction was intensified. Six cases with severe changes were selected. In all 6, the number of "units" was greatly reduced, so that " single discharges" were obtained. In this respect the picture was thus identical with that seen in moderate or severe neurogenic paresis. By way of comparison, the Table also shows the average of double determinations of six normal cases recorded from the same muscle, that is, five from the first interosseus and one from the peroneal muscle.

Dystrophia Myotonica.-In dystrophia myotonica, Cases 26-29, the electromyographic picture is the same as in progressive muscular dystrophy, apart from the specific myotonic changes analyzed by Lindsley and Curnen (1936), Denny-Brown and Nevin (1941), and Buchtal and Clemmesen (1941 b) in man, and by Brown and Harvey (1939) in myotonic goats.

Myasthenia Gravis.-It is also interesting to note that in myasthenia gravis, Cases $30-32$, the paretic
TABLE

\begin{tabular}{|c|c|c|c|c|c|}
\hline \multirow{2}{*}{$\begin{array}{c}\text { Case reference } \\
\text { and } \\
\text { muscle tested }\end{array}$} & \multicolumn{5}{|c|}{ Order of Recruitment } \\
\hline & 1 & 2 & 3 & 4 & 5 \\
\hline $\begin{array}{l}\text { Case } 11 \\
\text { inteross. } \\
\text { muscle }\end{array}$ & 50 & 100 & 100 & 450 & 300 \\
\hline $\begin{array}{l}\text { Case } 12 \\
\text { inteross.- } \\
\text { muscle }\end{array}$ & 50 & 100 & 200 & 250 & 800 \\
\hline $\begin{array}{l}\text { Case } 15 \\
\text { inteross. } \\
\text { muscle }\end{array}$ & 25 & 50 & 100 & 200 & 300 \\
\hline $\begin{array}{l}\text { Case } 16 \\
\text { ant. tibial } \\
\text { muscle }\end{array}$ & 50 & 100 & 300 & 600 & - \\
\hline $\begin{array}{l}\text { Case } 17 \\
\text { inteross. } \\
\text { muscle }\end{array}$ & 100 & 1,500 & 1,000 & 200 & - \\
\hline $\begin{array}{l}\text { Case } 18 \\
\text { inteross. } \\
\text { muscle }\end{array}$ & 50 & 150 & 200 & 2,000 & 600 \\
\hline $\begin{array}{ll}\text { Mean value } & . \\
\text { Range } & \ldots\end{array}$ & $\begin{array}{r}54 \\
25- \\
100\end{array}$ & $\begin{array}{c}408 \\
50 \\
.1,500\end{array}$ & $\begin{array}{l}316 \\
100 \\
1,000\end{array}$ & $\begin{array}{c}600 \\
200 \\
2,000\end{array}$ & $\begin{array}{r}500 \\
300 \\
800\end{array}$ \\
\hline $\begin{array}{l}6 \text { normal cases } \\
\text { Mean value .. } \\
\text { Range . } . .\end{array}$ & $\begin{array}{r}220 \\
100- \\
500\end{array}$ & $\begin{array}{c}480 \\
250- \\
800\end{array}$ & $\begin{array}{c}360 \\
50-\end{array}$ & $\begin{array}{c}490 \\
100- \\
800\end{array}$ & $\begin{array}{r}510 \\
100- \\
800\end{array}$ \\
\hline
\end{tabular}

Amplitude of spikes in order of recruitment in cases of late distal dystrophy and in normal cases. Amplitude in microvolts.

muscle shows an electromyographic picture in certain respects identical with that seen in progressive dystrophy. When the paresis is very pronounced, as exemplified by Fig. $10 \mathrm{a}$ and $\mathrm{b}$ from an almost paralytic biceps muscle (Case 30), the number of action potentials is only moderately reduced. The largest spike in the record can be followed on maximal voluntary contraction, against a background of smaller action potentials covering the base line (record b). As shown by Lindsley (1935), the amplitude of the spikes tends to diminish on continued contraction, as is also seen in record $a$ and $b$. When the muscle is paralytic (record $d$ from the masseter muscle of case 32), only a few active units, with a small amplitude, are left. Prostigmin rapidly restores the electromyogram to a more normal picture. As in muscular dystrophy, the extremely rapid action potentials are observed when the paresis is pronounced (Fig. 10c). 
Lesions OF THE LOWER MOTOR NeURONE

Reinnervation.-The electromyogram in reinnervation is in certain respects very similar to that seen in myopathy. This is exemplified by Fig. 11 from a case (No. 33) of localized neuritis of the shoulder girdle of the type described for example by Spillane (1943). The record was taken six months after the onset of the disease which clinically paralysed left deltoid muscle. There was no spontaneous fibrillation, but the insertion of the needle evoked a vigorous outburst of fibrillary action potentials, which died away after about ten seconds. Parts of the muscle were electrically silent on voluntary contraction. In other places small action potentials (Fig. 11a) were elicited, with a duration of 1 to 2 milliseconds. When the position of the needle was moved, action potentials of larger amplitude (record c), though with the same duration (record d), could be observed. Moreover, there were numerous polycyclic action potentials (records $\mathrm{b}$ and $\mathrm{e}$ ). These findings are in complete accord with those of Weddell and others (1944). Although numerous action potentials were brought into operation, the muscle was clinically paralysed. Thus; as in cases of dystrophy, we find for the most part rapid action potentials, representing less muscular power than the spikes in normal muscle. On the other hand numerous polycyclic action potentials and evidence of increased mechanical irritability do not appear in cases of muscular dystrophy.

From the clinical point of view, the somewhat similar pictures seen in muscular dystrophy and in reinnervation is of relatively little importance as the differential diagnosis generally follows from the case history.

Acute Peripheral Nerve Lesions.-The same remark applies to acute peripheral nerve lesions. In such cases the development of fibrillation, DennyBrown and Pennybacker, 1938, ten to eighteen days after denervation but not seen in reversible ischemic block, Weddell and others, 1944, is an extremely valuable sign. The voluntary action potentials are reduced in number, according to the degree of paresis. The duration and amplitude of the spikes usually lie within normal limits, as exemplified by Fig. 12 which was recorded from a clinically almost paralysed deltoid muscle (Case 34), examined 10 days after the onset of paresis. The duration of the single spike (record c) was 4 to 5 milliseconds, which is considerably greater than that observed in dystrophies. There was no fibrillation. Clinically, there was some suspicion of " psychogenic paresis." The frequency of discharge on maximal contraction, however, was 50 per second (records a and b), which shows that the patient, despite the great weakness of the muscle, had made a great effort to contract it.

Lesions of the Anterior Horn Cells. - In lesions of $\cong$ the anterior horn cells (amyotrophic lateral sclerosis, of poliomyelitis, syringomyelia, compression of the $\frac{\bar{C}}{0}$ spinal cord, etc.), and in certain cases of polyneuritis, peroneal muscular atrophy, inveterate peripheral $\stackrel{\infty}{O}^{\circ}$

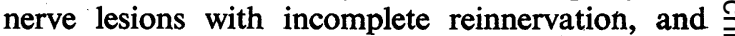
herniated nucleus pulposus, there is another type of electromyogram, of considerable importance in differential diagnosis.

Spontaneous fibrillation, according to my experience, may often be absent in this group. When paresis is slight no decrease in the number of action potentials on maximal voluntary contraction can be detected. They take up the whole base line and interfere with one another. However, the' spike on amplitude is often unduly large, at any rate as regards the third and the following action potentials. This valuable sign can be seen even in muscles which, on clinical examination, appear normal. $\mathrm{A}$ detailed study of the spike amplitude will be published later and a typical example may be sufficient here.

Even when the paresis is only moderate, the number of spikes is distinctly reduced and the $\mathrm{N}$ barely take up the base line. Reduction to this 0 extent thus occurs earlier than in muscular dys trophies with a similar degree of paresis. This $\square$ exemplified by Case 35 (Fig. 13), where the patient was suffering from peroneal muscular-atrophy.

Record 13a (calibration 500 microvolts) from the moderately paretic muscle of the right calf show that the number of action potentials is so reduced that they do not cover the base line on maximal contraction. The amplitude of the spike with which the voluntary contraction starts is 500 microvolts; the next 5 millivolts; the third 30 millivolts; the fourth and last 12 millivolts. If $₫$ we compare these amplitudes with the normal $\overrightarrow{\vec{D}}$ values in the Table, it will be found that they are much larger.

One should guard against mistaking action potentials evoked by the mechanical irritation of the needle for voluntary spikes. The mechanical irritability in denervated muscles is usually very $\dot{\sigma}$ marked, and a minor movement of the point of the 3 needle owing to muscular contraction often suffices $\bar{\varnothing}$ to elicit fibrillary action potentials or spikes with a greater amplitude and duration. Sometimes the 0 slight movement in the point of the needle that occurs when an adjacent unit contracts may suffice $\frac{D}{0}$ to evoke them. The repetitive action potentials of the fibrillary type (record 13d) or the larger ones $\widetilde{N}$ (record c) following the voluntary action potentials $N$ 
with a "latency time" of 20 to 100 milliseconds are most easily explained in this way. Repetition in the same unit as is innervated or in adjacent units, evoked by excitation of the action potential, is less probable. At any rate they should not be viewed as voluntary spikes. In that case they might easily be interpreted as spikes of the dystrophy type, seeing that their duration is usually shorter than normal.

\section{Discussion}

The most important new fact which has emerged from this electromyographic study is the early change in cases of primary myopathy consisting of the occurrence of an abnormally large number of very rapid action potentials with a duration of about 1 millisecond. These seem to be produced by the gradual shortening of the duration of the normal action potentials and presumably represent the final stage in this process.

The other changes in the electromyogram are an inconstant diminution in the amplitude of the spikes and a reduction in their number, which is clearly noticeable only when there is marked paresis of the muscle tested. As shown by Figs. 8 and 9, the number of action potentials remaining may be considerable, even in muscles which appear to be almost paralysed.

In such cases they represent much less muscular power than the average action potentials in normal muscle.

All these changes in the electromyogram are most easily explained on the assumption that the number of muscle fibres belonging to the "units" are gradually diminished by the pathological process. The " units" eventually are completely destroyed, but not all of them at the same time, and some are more resistant than others. Whether the process is connected mainly with the small spikes which start the voluntary contraction (Smith, 1934), and which were thought by Denny-Brown and Pennybacker (1938) to represent small units controlling a few muscle fibres, and have been shown by Kugelberg and Skoglund (1946) to be innervated by low-threshold nerve fibres, or whether the process is connected with the larger high-threshold spikes, could not be determined. The degenerative process, however, seems to be rather diffuse. When the number of spikes in the myogram is distinctly reduced, the remaining action potentials as a rule are also profoundly changed in that the duration is shortened both of the spikes activated at an early stage in contraction, and of those activated only after intense contraction.

The electromyographical picture of dystrophies seems to correspond with the histological changes described, for example, by Pappenheimer (1908),
Slauck (1921, 1932), and Bowden and Gutmann (1946).

It is not surprising that the electromyogram in muscular dystrophies should show changes very similar to those in cases of myasthenia gravis. As shown by Lindsley (1935), Harvey and Masland (1941), Harvey and others (1941), Harvey and Lilienthal (1941), the paresis is caused by a neuromuscular block. One muscle fibre after the other is blocked in the unit ; and, though this process is functional and reversible, its result will be essentially the same as if an increasing number of muscle fibres were put out of action in the unit by other more profound changes, as in muscular dystrophy.

It is, moreover, evident that the electromyographical picture in reinnervation must in some respects be identical with that in myopathies. All the muscle fibres in a "unit" cannot, of course, be reinnervated at the same time. The unit must, therefore, to begin with, consist of an abnormally small number of muscle fibres. Just as has been found to be the case in myopathies, the power of the muscle on maximal voluntary contraction 'will be poor despite the fact that many units are brought into operation. The individual action potentials tend to become more rapid and of lower voltage than the normal ones.

Myopathies affect individual muscle fibres, whereas lesions of the lower motor neurone affect whole " units." The destroyed unit belonging to one nerve fibre is put out of action at once and not gradually. If we have, say, 100 nerve fibres innervating 100 units, each composed of 100 muscle fibres, and destroy 90 per cent. of the nerve fibres, the number of units and the muscular power will be reduced to one tenth of the original. In myopathy, if 90 per cent. of the muscle cells had been uniformly destroyed in all units, the muscular power would likewise have been reduced to onetenth, but the number of functioning units would have remained unchanged.

A decrease in the number of spikes relative to the loss of muscular power occurs, therefore, much earlier in neurogenic than in myogenic lesions. However, as shown above, units are not always destroyed uniformly in cases of myopathy, though eventually there is a considerable reduction in their number, so that "single discharges" are obtained on maximal voluntary contraction. This occurs most often in localized forms of myopathy, which, clinically, may show great similarity to certain neurogenic diseases. In such cases the number of spikes in the electromyogram relatively to the degree. of paresis cannot serve as a basis for the differential diagnosis. Moreover, fibrillary twitchings 
are often absent in chronic neurogenic complaints such as those of the Charcot-Marie-Tooth type. For the differential diagnosis in such cases, we are thrown back upon the characteristic changes undergone by the action potentials with respect to duration and amplitude. This applies also to the recognition of the early stages of the motor neurone disease, when the paresis is so slight that the reduction in the number of action potentials is not yet observable. In this way a clear-cut differential diagnosis between myogenic and neurogenic lesions can be made.

\section{Summary}

The typical electromyographical sign of myopathies is the occurrence of spike potentials with an abnormally short duration. " Single discharge" waves also occur: these cannot, therefore, be regarded as pathognomonic for neurogenic lesions. In myopathies the spikes in " single discharges,"

i however, have an approximate duration of 1. millisecond, as against a duration of 5 to 10 milliseconds in neurogenic lesions (except in reinnervation).

1. In muscular dystrophy (6 cases of the proximal type, 4 of the Landouzy and Dejerine form, and 15 of a distal type with late onset), the earliest observable change, visible even in mild or moderately severe paresis, consists of a large number of spikes with a duration of about 1 millisecond. Intensification of the paresis is manifested by an increase in the relative number of rapid spikes, which at the final stage comprise all or most of the remaining action potentials. They represent a degree of strength which is much less than that of the average action potential in the normal muscle. The amplitude of the spikes may show an irregular reduction relative to normal.

$A$ reduction in the number of action potentials on voluntary contraction is clearly noticeable only when the paresis is marked. When the muscle is scarcely able to perform a movement in the joint, the spikes can be followed separately on maximal contraction. They then barely take up the whole base line on the electromyogram. When the paresis is still more pronounced, only a few action potentials remain, or the muscle may even be electrically silent.

The changes in the electrical activity are explained by the pathological process which throws one muscle fibre after another out of action. This proceeds fairly uniformly in the different muscle "units," though some of them are somewhat more resistant than others.
2. In dystrophia myotonica (4 cases) the voluntary action potentials in the atrophied muscle undergo essentially the same changes as in muscular dystrophy. The same remark applies to myasthenia gravis ( 3 cases), where the muscle fibres are put out of action in the "units" by a functional and reversible process.

3. With special regard to differential diagnosis, certain characteristic changes in the electromyogram in lesions of the lower motor neurone are discussed, and contrasted with the electrical changes in myopathy.

References
Adrian, E. D., and Bronk, D. W. (1929). J. Physiol., 67, 119.

Bowden, R. E. M., and Gutmann, E. (1946). Arch. Neurol. Psychiat., Chicago, 56, 1.

Brown, G. L., and Harvey, A. M. (1939). Brain., 62, is

341.
Buchthal, F., and Clemmesen, S. (1941 a). Acta neurol. psychiatr., 16, 143.

- - (1941 b). Ibid., 16, 389.

Denny-Brown, D., and Pennybacker, J. B. . (1938). Brain, 61, 311.

, and Nevin, S. (1941). Ibid., 64, 1.

Gilson, A. S. Jr., and Mills, W. B. (1941). Amer. J. $\vec{\circ}$ Physiol., 133, 658.

Harvey, A. M., and Masland, R. L. (1941). .Johe Hopk. Hosp. Bull., 69, 1. - , Lilienthal, J. L., and Talbot, S. A. (1941). Ibi⿻ 69, 547.

Hoefer, P. F. A., and Putnam, T. J. (1939). Arch. Neurg. Ibid. 69, Psychiat., Chicago, 42, 201.

Kugelberg, E., and Skoglund, C. R. (1946). J. Neurephysiol., 9, 399.

Landouzy, L., and Déjerine, J. (1884). Paris, 98, 53.

P. (1886). C. R. Soc. Biol., Paris, 8s, 3, 478.

Lindsley, D. B. (1935). Brain., 58, 470.

, and Curnen, E. C. (1936). Arch. Neurol. Psychiat., Chicago, 35, 253.

Milhorat, A. T., and Wolff, H. G. (1943). Ibid., 49, 655.气

Pappenheimer, A. M. (1908). Beitr. path. Anat. allg.气 Path., 44, 430.

Seyffarth, H. (1938). Acta neurol. psychiatr., 13, 297.

- (1940). Skr. norske Videnskapsakad., 4, suppl. XII.

Slauck, A. (1921). Z. Neurol. Psychiat., 71, 352. (1932). In Bumke, O., and Foerster, O. : "Hand buch der Neurologie." Berlin : Julius Springer. Vol. 16, p. 412.

Smith, O. C. (1934). Amer. J. Physiol., 108, 629.

Snodgrass, T. M. and Sperry, R. W. (1941). Amer. Physiol., 133, 455.

Spillane, J. D. (1943). Lancet, 2, 532.

Weddell, G., Feinstein, B., and Pattle, R. E. (1944) Brain, 67, 178.

Welander, L. (1946). $\quad$ Nord. Med., 29, 618. 


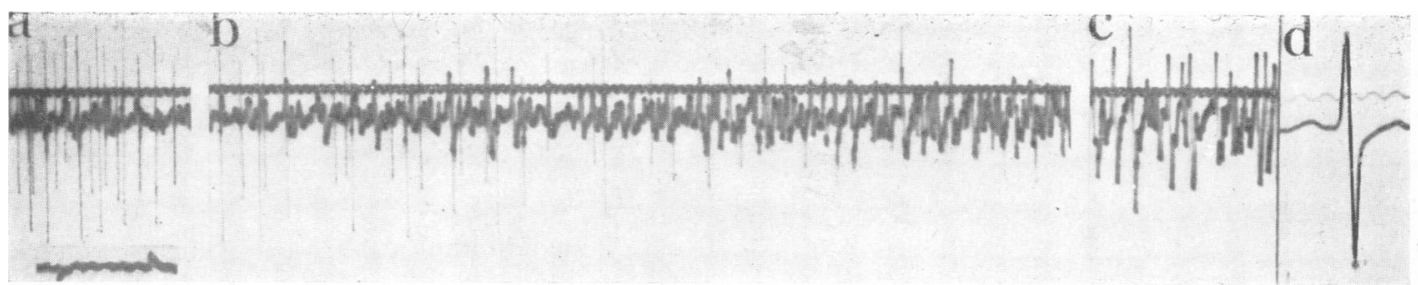

FIG 1.-Muscular dystrophy (Case 1). Records from the first interosseus muscle. a.-Maximal voluntary contraction with properly placed needle electrode, showing perfect isolation of the action potentials which are reduced in number. $b$ and $c$.-Needle is gently shifted a few $\mathrm{mm}$. as contraction is going on. The distinct spikes are replaced by interfering activity of no diagnostic value. $d$.-Detail picture of a typical dystrophy spike with a duration of about 1 millisecond.

In this and the following figures: calibration 100 microvolt (except Fig. 15a). Time 1/100 sec. In detail pictures of single spikes, time $1 / 1,000 \mathrm{sec}$.
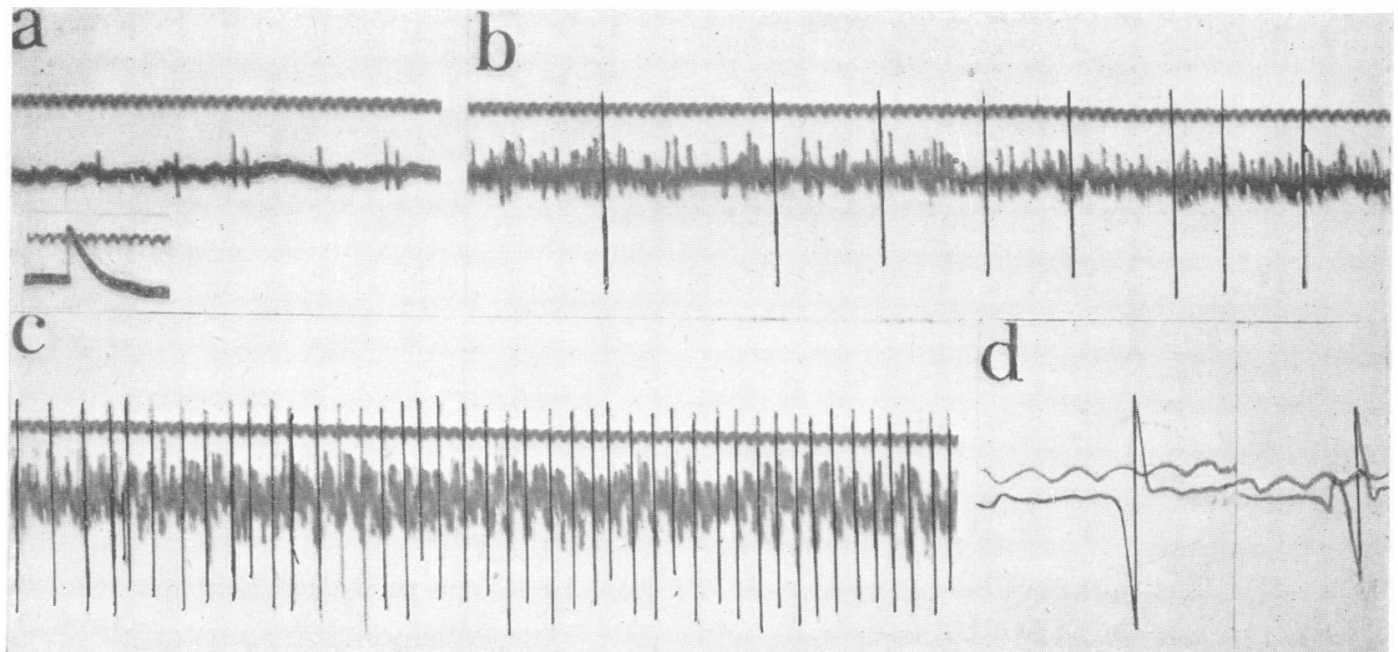

FIG. 2.-Muscular dystrophy proximal form (Case 5). Records from the greatly atrophied biceps muscle. $a, b$, and $c$.- Showing the activity of a voluntary contraction gradually increasing in strength. In $d$ a detail picture of the largest action potential seen in $b$ and $c$.

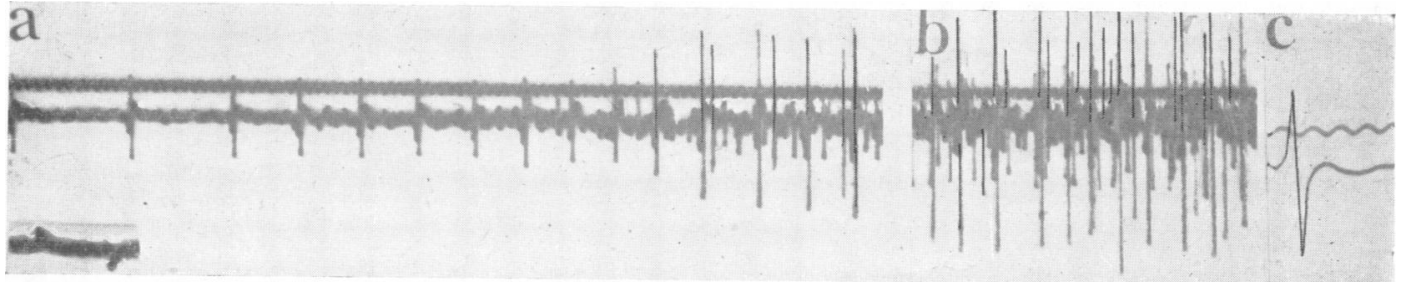

Fig. 3.-Muscular dystrophy (Case 4). Records from the highly paretic quadriceps muscle.

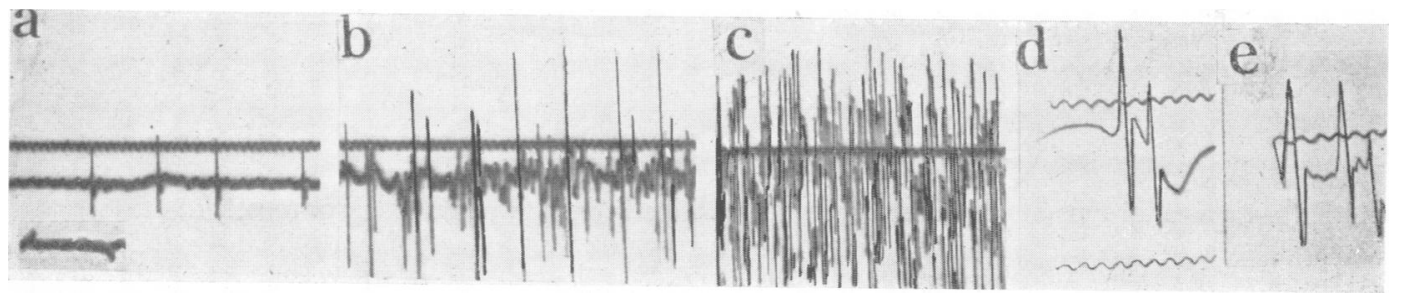

FIG. 4.-Muscular dystrophy (Case 2). Records from the moderately paretic quadriceps muscle. 
a $\quad b$

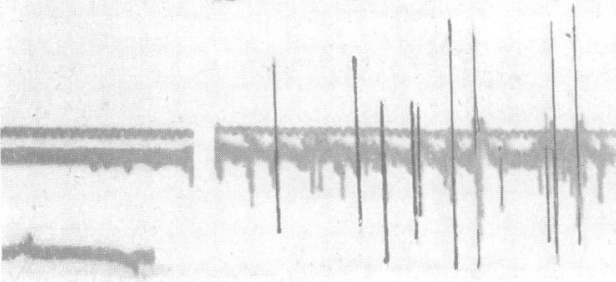

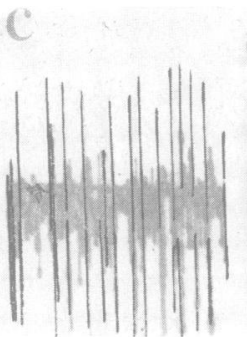
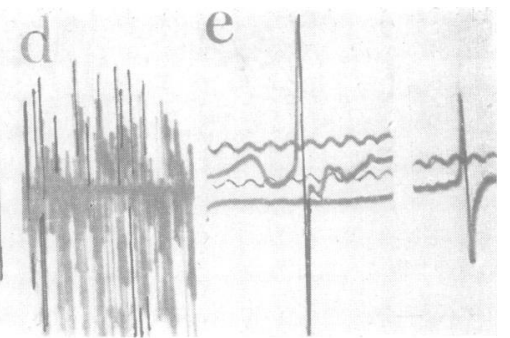

FI(i. 5.--Muscular dystrophy (Case 3). Records from the slightly paretic biceps muscle.

a

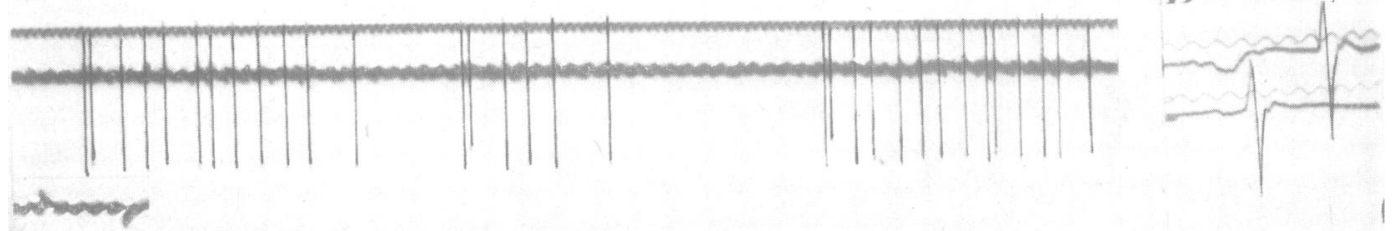

C

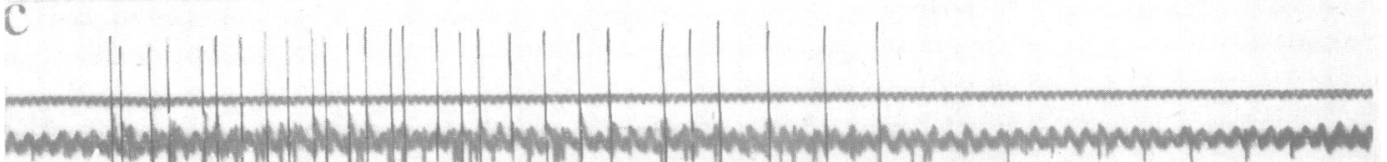

tap

FIG. 6.--Iate distal type of myopathy (Case 12). Records from the clinically paralytic anterior tibial muscle.
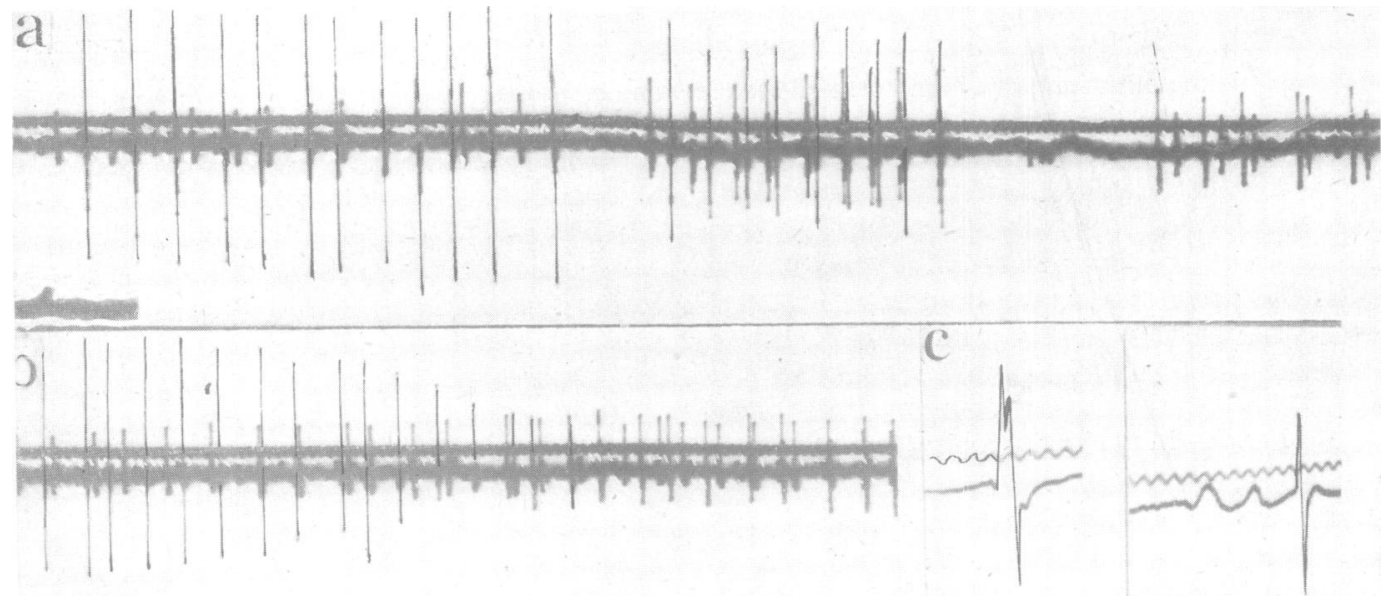

Fici. 7. -Late distal type of myopathy (Case 14). Records from the short extensor muscle of the toes. 

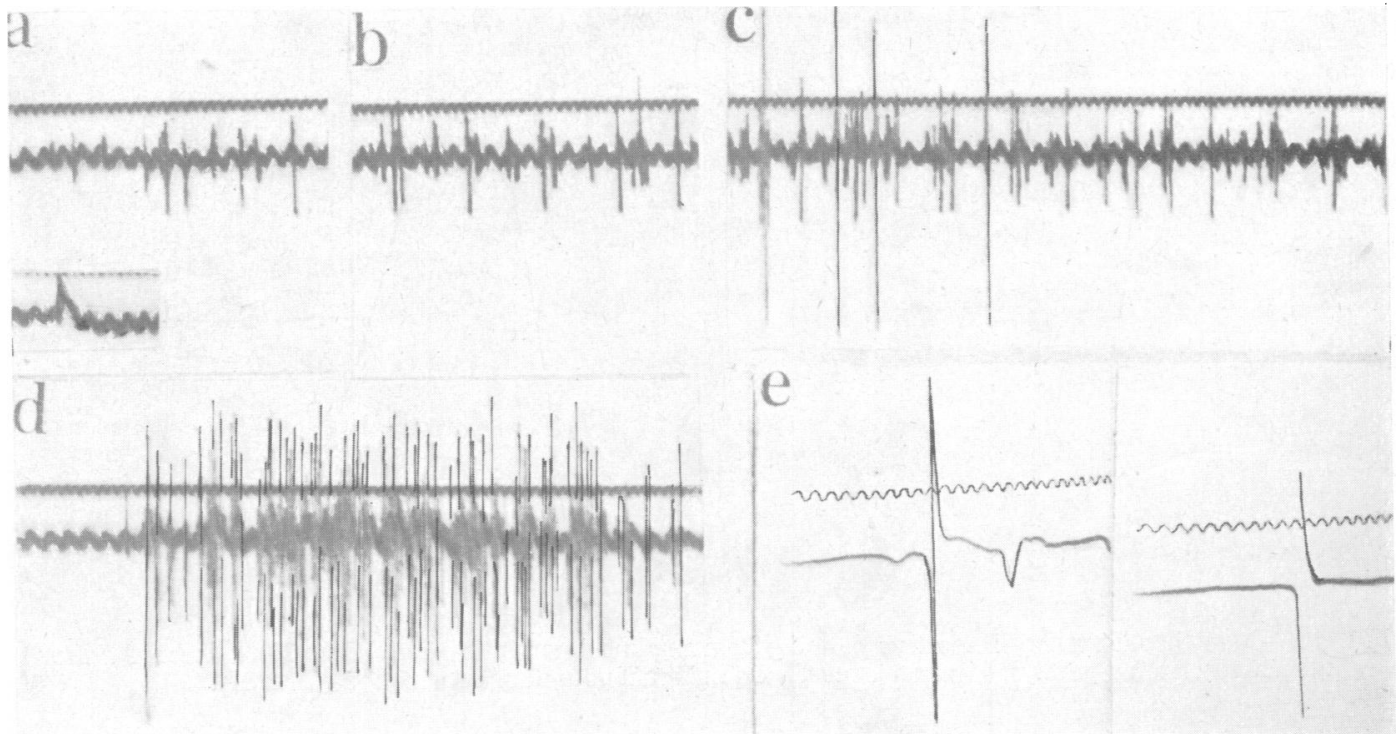

FIG. 8.-Late distal type of myopathy Case 12). Records from the first interosseus muscle which could barely move the second finger.

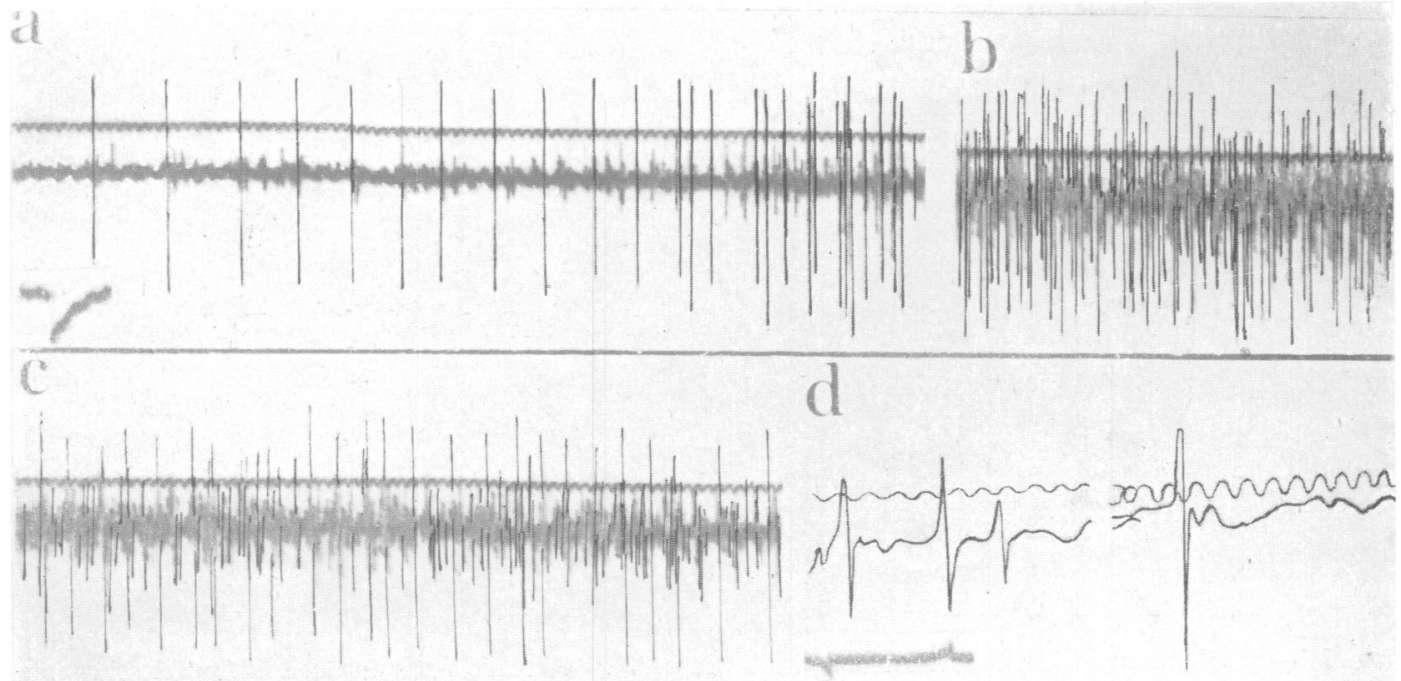

FIG. 9.- - Late distal type of myopathy (Case 15). Records from the highly paretic first interosseus muscle. 


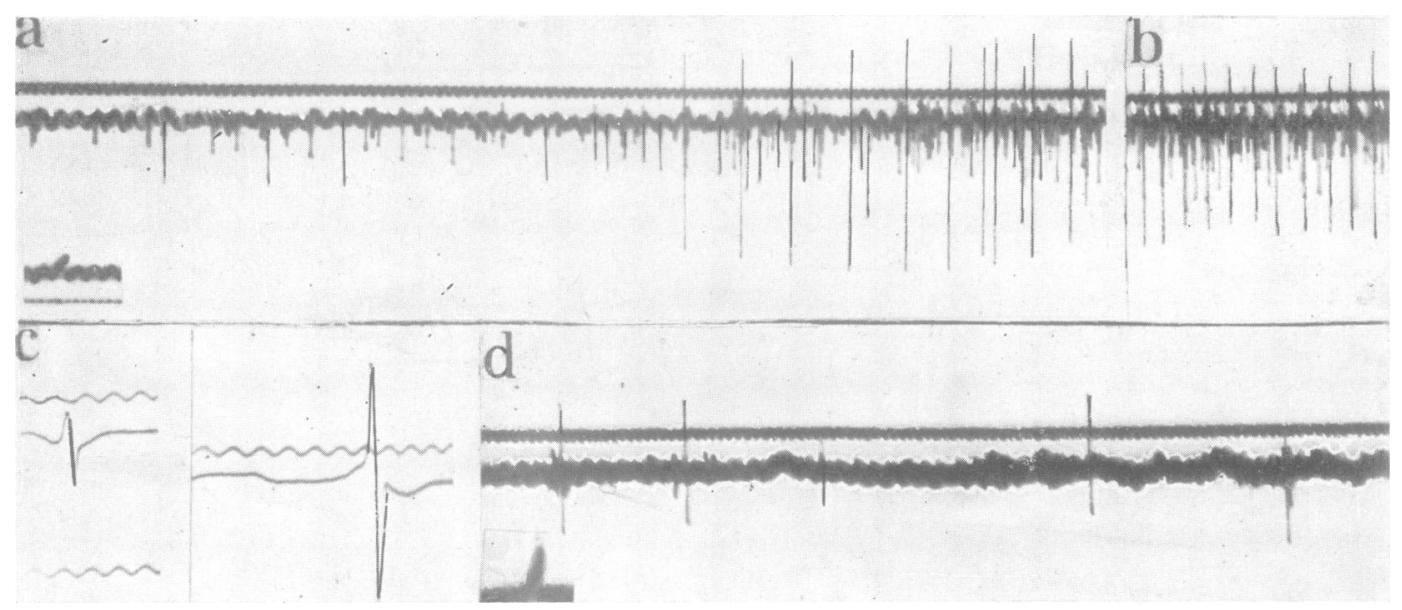

FIG. 10.-Myasthenia gravis. Records $a, b$, and $c$ from an almost paralytic biceps (Case 30). Record $d$ shows the activity from a paralysed masseter muscle on maximal voluntary contraction (Case 32 ).

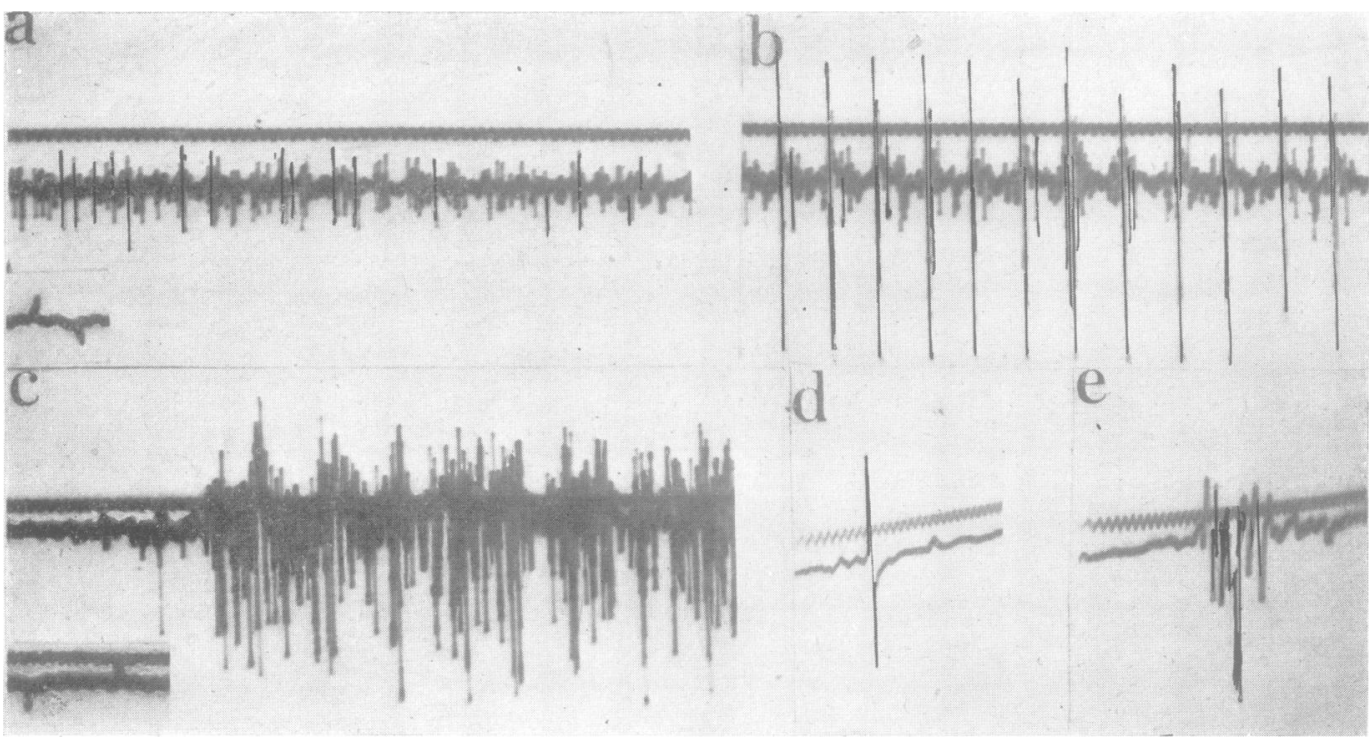

FIG. 11.-Localized neuritis of the shoulder girdle (Case 33). Records from the deltoid muscle showing reinnervation activity. 


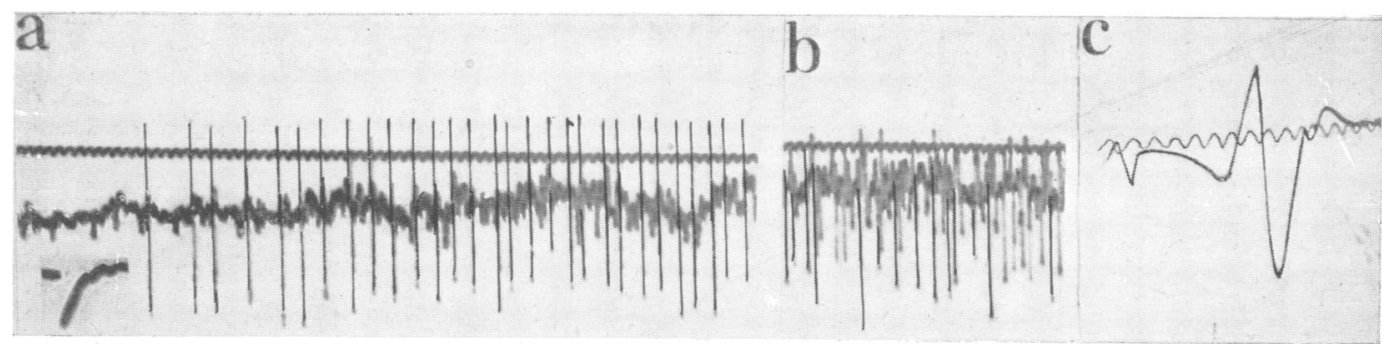

FIG. 12.-Acute peripheral nerve lesion (Case 34). Records from the clinically almost completely paralysed deltoid muscle.

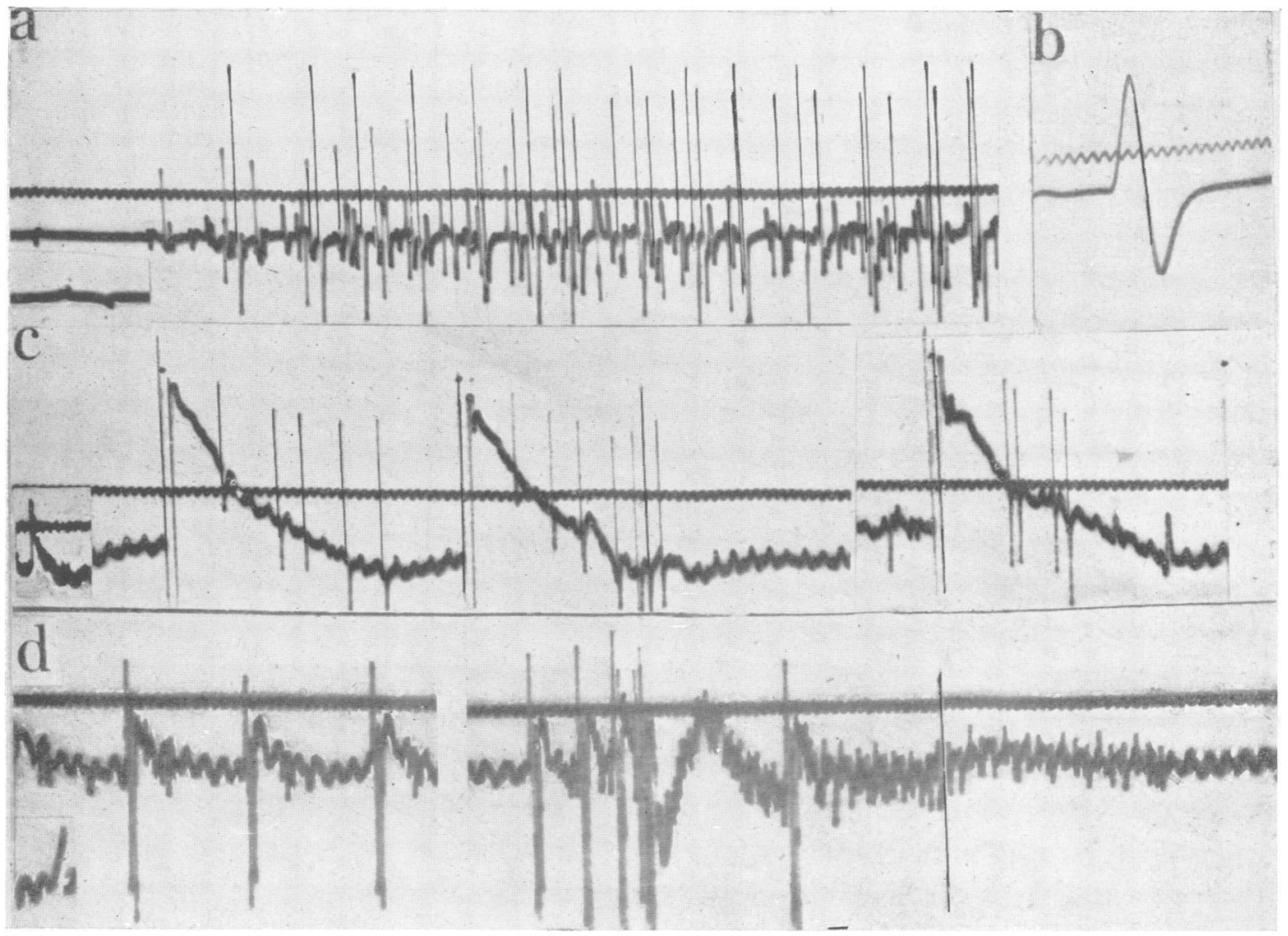

FIG. 13.-Peroneal muscular atrophy (Cases 35 and 36). Records from the moderately paretic calf muscle. a.-Calibration 500 microvolts. Shows a voluntary contraction increasing to maximal strength. The action potentials are reduced in number and of pathological large amplitude. b.-Detail picture of one action potential. c.-Larger amplification (calibration 100 microvolts). The voluntary spikes which are too large to be registered correctly at this amplification are followed by repetitive spikes of shorter duration and smaller amplitude. $d$. (Case 36). - The voluntary action potentials are followed by repetitive spikes of the fibrillary type. The base line is somewhat disturbed by 50 cycles per second alternating current. 